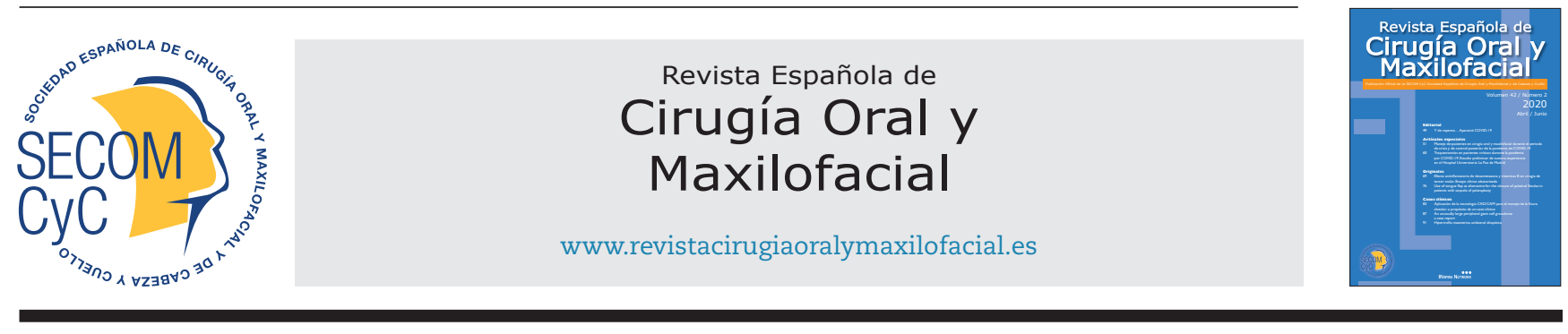

\title{
Editorial
}

\section{Y de repente... Apareció COVID-19}

Hasta hace poco más de 4 meses, como profesionales de la Cirugía Oral y Maxilofacial, nuestras preocupaciones se centraban en ofrecer los mejores tratamientos para nuestros pacientes, en establecer medidas para solucionar las listas de espera, en la adquisición de nuevos materiales para nuestros centros, en la comunicación de resultados por medio de publicaciones en revistas científicas y en la elaboración de ponencias y comunicaciones para congresos y reuniones de la Especialidad. Así era hasta que apareció "el bicho", el coronavirus COVID-19, que dio al traste con nuestro particular "paraíso", ese mundo anterior a la crisis donde trabajábamos tanto y tan rápido, y donde nos encaramábamos a lomos de ese "tigre" en movimiento que nos impedía reflexionar acerca de nuestra actividad del día a día. Y de repente, todo se detuvo... Empezó un confinamiento medieval, todos en casa, sin paliativos.

Escuchamos en muchos medios acerca de otras pandemias históricas u otras crisis nacionales, pero como esta ninguna, entre otras cosas porque es la que nos está tocando vivir. Un tercio de la humanidad ha estado confinada y se ha levantado un Leviatán sanitario que ha dejado meridianamente claro que nuestras autoridades gubernamentales han actuado tarde, mal y sin los medios necesarios y requeridos por los sanitarios para afrontar con seguridad esta crisis. Tiempo habrá de pedir responsabilidades de toda índole, pero la textura de la crisis nos indica que no éramos tan sólidos antes, y que seremos, por tiempo indefinido, más frágiles de lo que hubiéramos creído. De entrada, vamos a sufrir una recesión global que tendrá unas repercusiones a corto y largo plazo. Pero los pacientes con patología maxilofacial van a seguir existiendo y hay que pensar en ellos.

El primer objetivo debe ser la protección de nuestros pacientes, pero al mismo tiempo la nuestra propia y la de nuestra familia. La Junta Directiva de la Sociedad Española de Cirugía Oral y Maxilofacial y de Cirugía de Cabeza y Cuello (SECOM-CyC) elaboró unos comunicados donde se establecían unas recomendaciones iniciales (14 de marzo de 2020), días más tarde modificadas muy parcialmente, y una serie de recomendaciones para la actividad en la "desescalada" o "desconfinamiento", enviadas a sus socios el día 29 de abril de 2020. Todas ellas se basaron en una evidencia científica a priori muy lejos de la Medicina Basada en las Existencias, que nuestras autoridades nos querían exponer como la única salida a esta crisis. Por otro lado, en noviembre de 2019 se diseñó un programa de "Noches Científicas con los Expertos" que, como cualquier conferencia por la web durante esta pandemia, se ha convertido en un arma de relación y formación muy potente. Por ahora la asistencia ha sido masiva en comparación con otros eventos similares. En este mismo número, la Revista Española de Cirugía Oral y Maxilofacial, como órgano de expresión de nuestra Especialidad, publica un artículo especial, a modo de guía, acerca de las recomendaciones que ha establecido la SECOMCyC para el manejo del paciente COVID-19 en nuestra área.

Superaremos esta crisis, pero todo va a ser diferente durante mucho tiempo. De entrada, vamos a estar amenazados por nuevos confinamientos totales o parciales y tendremos que pensar en ello en la gestión de nuestro trabajo diario para establecer planes de contingencia que aborden el peor de los escenarios. Seguiremos trabajando, pero con menos pacientes si se siguen las normas de lógico cumplimiento. Nuestra Especialidad está muy bien posicionada porque, entre otras cosas, siempre se ha movido en una necesidad de mantener la esterilidad y medidas de seguridad, incluso para realizar intervenciones que pueden ser

\footnotetext{
*Autor para correspondencia:

Correo electrónico: fmonje@oralmaxilofacial.com (Florencio Monje Gil).
}

DOI: $10.20986 /$ recom.2020.1155/2020

1130-0558/@ 2020 SECOM. Publicado por Inspira Network. Este es un artículo Open Access bajo la licencia CC BY-NC-ND (http:// creativecommons.org/licenses/by-nc-nd/4.0/). 
consideradas menores. Por lo tanto, la adaptación a estas circunstancias no nos supone un esfuerzo añadido enorme en comparación con otros sanitarios. Aun así, el coste económico institucional, empresarial o personal para esta adaptación a las normas va ser considerable y demoledor, pero habrá que ir haciéndolo antes de la ansiada "normalidad". La primera idea que, de forma enérgica, hay que trasladar a la sociedad es que aquellas instalaciones que puedan ser manejadas por nuestros especialistas de forma directa son lugares seguros en los que se contempla de forma prioritaria la seguridad de nuestros pacientes. Esta tarea es fundamental, pues los pacientes serán los primeros en exigírnoslo. Como botón de muestra está el hecho de que durante la pandemia ha disminuido la asistencia a los servicios de urgencia, no porque no hayan sucedido los episodios que habitualmente eran atendidos en ellos sino por el hecho de que la población, en base a las noticias recibidas por los medios de comunicación, ha considerado cualquier centro sanitario como un lugar propicio para un potencial contagio. Muchos de nuestros compañeros han tenido urgencias derivadas de postoperatorios de nuestros tratamientos durante el confinamiento que han preferido solucionar por teléfono para evitar que el paciente tuviera que trasladarse al centro sanitario.

Hemos de acostumbrarnos, por el rigor de toda la normativa, a ser más "slow surgery" (no vamos a seguir moviéndonos en un entorno de multitarea ni operar varios pacientes en la misma franja horaria) y más integrales en el tratamiento. En numerosas ocasiones, nuestro tratamiento es el esencial, pero en otras somos una parte más de la cadena, que debe ser revisada intentando instaurar un protocolo donde nosotros controlemos el tratamiento global de ese paciente o de esa patología. Asimismo, la telemedicina puede paliar los problemas asistenciales y esta crisis ha demostrado que es un arma interesante. Personalmente, no considero que la telemedicina sea la panacea, pues nuestra profesión es clínica y personal, pero en muchas ocasiones puede ayudarnos en la valoración inicial de pacientes que distan mucho de nuestros centros (para luego pedir pruebas complementarias) o en revisiones de patologías que no necesitan ningún tipo de exploración específica.

En este entorno de miedo, de precauciones, de evaluación de rentabilidades, no puede faltar el sello identificador de nuestra especialidad: la calidad y la excelencia. Ha sido una de las causas que, a pesar de los ataques o críticas que hemos recibido, han valido para identificarnos como profesionales capacitados y muy preparados para satisfacer, en tiempo y forma, los problemas derivados de la patología oral y maxilofacial y de cabeza y cuello de los pacientes. Por ello, y en esta amenaza de nuevos contagios y nuevos confinamientos, deberíamos ir pensando en la adaptación de todas las instalaciones a situaciones análogas futuras: la presión positiva o negativa de los quirófanos, las aplicaciones de gestión de pacientes, los sistemas de protección simples, etc. Quizás esta crisis nos permita acelerar cambios que, de otra manera, se afrontarían en un periodo mucho mayor.

En este ambiente de pesimismo y de ocultación de información, cuando no de flagrante retorcimiento de la verdad en los medios de comunicación de masas, hay una obligación de establecer una mirada positiva y optimista. Cuando salgamos de esta crisis deberíamos homenajear a todos los sanitarios, pero en especial a los residentes de todas las especialidades, que han cargado a sus espaldas el peso de la actividad asistencial y de urgencias durante esta crisis. Los de nuestra especialidad son parte de ellos. La Cirugía Oral y Maxilofacial es una especialidad médica joven todavía, bien posicionada en Universidades y en I+D (aunque mejorable), y con áreas de actuación muy específicas que necesitan ser tratadas; además deberemos establecer claramente la importante misión de la docencia y formación de nuestros residentes, el futuro de nuestra especialidad, al fin y al cabo, que no puede verse disminuida. Esta crisis sanitaria y económica debe convertirse en el acicate para mejorar como profesionales en nuestro quehacer diario y como ciudadanos de nuestra sociedad.

Por último, pero en primer lugar, desde la Junta Directiva de SECOM-CyC y desde el Comité Editorial de la Revista Española de Cirugía Oral y Maxilofacial queremos expresar nuestro más sentido pésame a todos aquellos de nuestros socios que hayan perdido algún familiar en esta tremenda pandemia, y animamos a la sociedad española en su conjunto a luchar unida para superar las enormes repercusiones de índole sanitario, económico y social que nos depararán los próximos meses. A pesar de todo, somos una gran nación.

Florencio Monje Gila y Raúl González García ${ }^{b}$ aPresidente de la Sociedad Española de Cirugía Oral y Maxilofacial y de Cirugía de Cabeza y Cuello. ${ }^{b}$ Director de la Revista Española de Cirugía Oral y Maxilofacial 